\title{
Relevanz von Empathie für dyadische Beziehungen - Über ein unterschätztes Konstrukt in der Coach-Klienten-Interaktion
}

\author{
Theresa Will ${ }^{1}$ - Simone Kauffeld ${ }^{1}$
}

Online publiziert: 19. Juni 2018

(c) Der/die Autor(en) 2018

\section{Zusammenfassung}

Empathie gilt als eine der wichtigsten Einflussfaktoren in dyadischen Beziehungen. Im Coachingkontext wird Empathie zwar häufig als wichtige Einflussvariable erwähnt, bisher wurde das Konstrukt jedoch nur sehr wenig beforscht. Um das Konstrukt Empathie mit seinen zwei Facetten (emotionale und kognitive Empathie) und die Bedeutung für den Coachingkontext besser einordnen zu können, wird in diesem Beitrag ein Überblick über Definitionen und potenzielle Möglichkeiten zur Messung gegeben. Hierbei orientieren wir uns an der Psychotherapieforschung, die sich seit vielen Jahren mit der Definition und Erfassung von Empathie beschäftigt. Daran anschließend wird die aktuell noch geringe Anzahl an empirischen Studien mit dem Fokus auf Empathie im Coaching vorgestellt. Die Ergebnisse der wenigen publizierten Studien unterstreichen die Relevanz von Empathie für den Coachingkontext. Zwischen emotionaler und kognitiver Empathie wird dabei meist nicht explizit unterschieden. Empfehlungen für zukünftige Forschung werden ebenso wie praktische Implikationen für praktizierende Coaches basierend auf den bisherigen Forschungserkenntnissen abgeleitet.

Schlüsselwörter Empathie $\cdot$ Emotionale Empathie $\cdot$ Kognitive Empathie $\cdot$ Coach-Klienten-Beziehung $\cdot$ Coaching Prozess

\section{Relevance of Empathy for Dyadic Relations - About an Underestimated Construct in the Coach-Client-Interaction}

\begin{abstract}
Empathy is seen as one of the most important influencing variables in dyadic relationships. Within the coaching context, empathy is often named as being an important influencing variable. Thus far, however, there is only little research on the construct. In order to classify the construct of empathy with its two facets (emotional and cognitive empathy) and its relevance for the coaching context, an overview of definitions and potential ways of measuring empathy will be given in this contribution. At this juncture, we orientate on the research from the field of psychotherapy since this field has focused on the definitions and measurements of empathy for a longer time. Next, we present the amount of empirical studies, which is currently still sparse, by focusing on empathy in coaching. The results of the publicized research underline the relevance of empathy for the coaching context. However, a differentiation between emotional and cognitive empathy can mostly not be found. Future research ideas and practical implications for coaches working in this setting, will be deducted.
\end{abstract}

Keywords Empathy $\cdot$ Emotional empathy $\cdot$ Cognitive empathy $\cdot$ Coach-client-relationship $\cdot$ Coaching process

Publisher's Note Springer Nature remains neutral with regard to jurisdictional claims in published maps and institutional affiliations.

Theresa Will

t.will@tu-bs.de, theresa.will@de.ey.com

1 Lehrstuhl für Arbeits-, Organisations- und

Sozialpsychologie, Technische Universität Braunschweig,

Spielmannstr. 19, 38106 Braunschweig, Deutschland 


\section{Einleitung}

„To sense the client's private world as if it were your own, but without ever losing the ,as if' quality - this is empathy, and this seems essential“" (Rogers 2007, S. 243)

Empathie wird als eine der fundamentalen Fähigkeiten für diejenigen verstanden, die in Beratungskontexten arbeiten. Auch die psychologische Forschung widmet sich seit vielen Jahren der Bedeutung von Empathie und beforscht ihre Relevanz (Burns und Nolen-Hoeksema 1992; Lambert und Barley 2001). Laut Rogers (u. a. 1951, 1957) zählt Empathie neben Wertschätzung und Kongruenz zu den Kernfähigkeiten, die eine Therapeuten-Klienten-Beziehung ausmachen.

Das Konstrukt Empathie wird seit mehreren Jahrzehnten als eine der wichtigsten Variablen für die Förderung der Beziehungsqualität zwischen Therapeut und Klient (z.B. Kurtz und Grummon 1972) angesehen, da Empathie unter anderem dabei hilft, dass die effektive Kommunikation gefördert (Nicolai et al. 2007) und der Beziehungsaufbau zwischen Individuen erleichtert werden kann (z.B. Lambert und Barley 2001; Norcross und Wampold 2011). Empathisches Reagieren hängt außerdem positiv mit prosozialem Verhalten zusammen (Miller und Eisenberg 1988) und kann positive Ergebnisse einer Therapie verstärken (Anderson et al. 2009; Horvath et al. 2011).

Für den Erfolg von Coaching wird die Relevanz von Empathie ebenfalls vermehrt hervorgehoben (Bluckert 2005; Feldman und Lankau 2005). Empathie gilt als eine der wichtigsten Einflussfaktoren für funktionierende CoachKlienten-Beziehungen. Besonders Klienten betonen die Wichtigkeit von gezeigter Empathie durch den Coach (Hall et al. 1999). Obwohl die Bedeutung der Empathie für die Interaktion zwischen Coach und Klient hervorgehoben wird, gibt es erst seit wenigen Jahren vereinzelte Studien, die sich mit der Thematik von Empathie im Coaching befassen.

Um das Konstrukt Empathie mit seinen zwei Facetten besser verstehen und die Relevanz für den Bereich Coaching einordnen zu können, soll der vorliegende Artikel einen Überblick über den aktuellen Definitions-, Messungsund Forschungsstand von Empathie geben. Dabei werden die beiden Facetten der Empathie (emotional und kognitiv) vorgestellt. Da die Psychotherapieforschung in diesem Feld dem der Coachingforschung weit voraus ist (Greif 2013), wird vornehmlich auf diesen Bereich Bezug genommen. Daran anschließend wird die Bedeutung von gezeigter Coachempathie für die Coachingforschung dargestellt. Es wird gezeigt, welche Form der Coachempathie besonders für die Beziehung zwischen Coach und Klient und die damit einhergehende Interaktion von hoher Bedeutung ist. Der aktuelle Forschungsstand wird abschließend diskutiert und zukünftige Forschungsideen sowie potentielle Ansatzpunkte für Praktiker abgeleitet.

\section{Definition(-en) von Empathie}

Bereits vor 80 Jahren beklagte Theodor Reik (1948), dass das Wort Empathie mal eine Sache bedeutet, manchmal eine andere, bis es nunmehr gar nichts bedeutet. Auch gegenwärtig besteht immer noch kein allgemeiner Konsens darüber, wie Empathie forschungsübergreifend definiert werden sollte. Sowohl früher (Smith 1759; Spencer 1870) als auch heute (z.B. Reniers et al. 2011) ist hingegen unbestritten, dass Empathie auf einer emotionalen sowie einer kognitiven Facette gründet, und somit eine zwei-Faktoren-Struktur aufweist.

Bei der emotionalen Empathie wird davon ausgegangen, dass das Individuum denselben Gefühlszustand erreicht, wie das beobachtete Gegenüber (Eisenberg und Miller 1987). Emotionale Empathie kann definiert werden als eine individuelle emotionale Antwort auf ein wahrgenommenes emotionales Erlebnis von anderen (Mehrabian et al. 1988). Beispielsweise wird Traurigkeit empfunden, wenn man ein weinendes Kind sieht oder es wird ein Glücksgefühl wahrgenommen, wenn sich ein Gegenüber augenscheinlich freut. Durch Gefühlsansteckung passt sich beispielsweise als äußerer Gefühlsausdruck die eigene Mimik der beobachteten Situation (häufig unbewusst) an. Dieses Phänomen der äußeren Mimik Anpassung findet sich bei dem Konstrukt der emotionalen Ansteckung (z. B. Hatfield et al. 2009). Unter emotionaler Ansteckung wird beispielsweise verstanden, dass in einer Konversation Menschen automatisch und kontinuierlich ihre Mimik oder aber auch ihre Tonlage, ihre Wortwahl oder sogar die Gestik an die eines Gegenübers anpassen (Hatfield et al. 2009). Als Resultat dieser Reflektion, ergeben sich beim Menschen ähnliche Gefühle als eine Art der Feedbackreaktion. Dies bedeutet, dass die Menschen die Gefühle des Gegenübers aufnehmen und etwas sehr ähnliches empfinden (Hatfield et al. 2009). Die Fähigkeit sich in die Gefühle anderer Menschen hineinzufühlen (emotionale Empathie) kann demnach als Folge des Prozesses der emotionale Ansteckung angesehen werden (Hatfield et al. 2009). Die emotionale Empathie beschreibt schlussendlich eine affektive Reaktion auf eine beobachtete Situation (Dadds et al. 2008). Zusammenfassend steht bei der emotionalen Empathie also das unbewusste und wenig steuerbare Nachempfinden einer wahrgenommenen Emotion im Vordergrund.

Der emotionalen Empathie gegenüber steht das Konstrukt der kognitiven Empathie. Die kognitive Empathie ist dadurch charakterisiert, dass die Sichtweise des Gegenübers gedanklich nachvollzogen werden kann (z.B. BaronCohen und Wheelwright 2004; Parra 2013). Ein Individu- 
um versucht sich aktiv in das Gegenüber hineinzuversetzen und eine kognitive Perspektivübernahme anzunehmen (Smith 2006). Kognitive Empathie bedeutet somit: Verstehen, warum jemand so handelt, wie er handelt. Preston und Hofelich (2011) definieren die kognitive Empathie als ein Verstehen eines Gegenübers durch eine Anpassung der eigenen Sichtweise. Für den Coachingkontext erscheint die kognitive Empathie wichtiger zu sein als die emotionale, da es darum geht als Coach dem Klienten zu zeigen, dass man ihn versteht, um ihn bei der Lösungsfindung gut unterstützen zu können und nicht darum, dass man seine Situation nachempfindet (z.B. Bluckert 2005; Hall und Bernieri 2001; Will et al. 2016).

\section{Empathie im Prozess}

Eine für den Coachingkontext formulierte neuere Definition für kognitive Empathie beschreibt die Beziehung zwischen Coach und Klient als eine Fähigkeit durch die ein Coach in der Lage ist sich den internen Zustand eines Klienten vorzustellen und dadurch versucht den Gedanken des Klienten zu verstehen (Will et al. 2016). Empathie im Coaching wird hier erstmals innerhalb einer Interaktion in ein Prozessmodell, basierend auf dem Empathie Zirkel (u.a. Barett-Lennard 1981) eingegliedert. Das ursprünglich für den therapeutischen Hintergrund entwickelte Modell kann ebenfalls auf den Coachingkontext übertragen werden und findet sich in den einzelnen Sequenzen auch in einer Cochinginteraktion wieder (Will et al. 2016) (für einen Überblick siehe Abb. 1). Das Prozessmodell besteht aus vier Phasen. So folgt aus der empathischen Wahrnehmung des Coaches eine empathische Kommunikation, die durch das Verhalten des Coaches ausgedrückt wird. Als Resultat auf das vom Coach gezeigte empathische Verhalten wird zunächst die Wahrnehmung durch den Klienten festgestellt und dann eine Reaktion auf das empathische Coachverhalten beobachtet.

Da Empathie sowohl im therapeutischen als auch im Coachingkontext für die Beziehungsgestaltung wichtig zu sein scheint, soll kurz dargestellt werden, welche Gemeinsamkeiten, aber auch welche Unterschiede zwischen Coach und Klient beziehungsweise Therapeut und Patient in der Beziehung festgehalten werden können. Im Coaching, aber auch in der Psychotherapie handelt es sich um Interaktionen, in denen eine wichtige Arbeitsbeziehung zwischen Coach und Klient sowie Therapeut und Patient im Fokus stehen (Ianiro und Kauffeld 2014). Das Anliegen des Klienten oder Patienten soll bearbeitet werden mit dem Ziel eine Verhaltensänderung hervorzurufen (Bluckert 2005a). Wichtig hierbei ist jedoch, dass es nicht um eine ausschließlich unilaterale Beziehung, geformt durch den Therapeuten oder Coach geht, sondern es eine Beeinflussung durch den

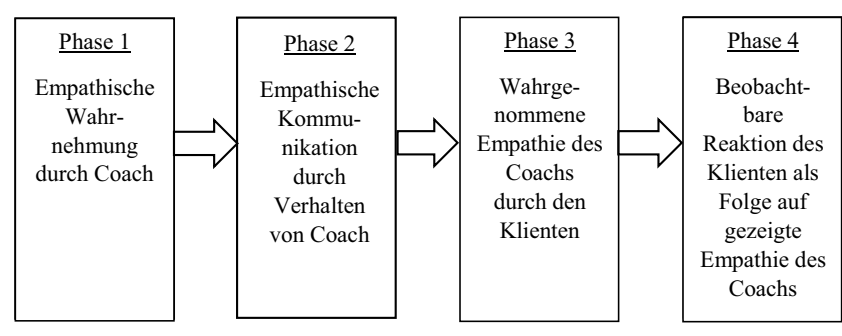

Abb. 1 Der Empathie Zirkel (basierend auf Barrett-Lennard 1981; für den Coachingkontext angepasst von Will et al. 2016)

Klienten gibt. Der Wunsch Veränderung beim Klienten oder Patienten hervorzurufen, aber auch die kognitiven und emotionalen Reaktionen des Klienten verstehen zu wollen sind Bestandteile beider Beziehungen. Neben dem Ausdrücken von Vertrauen, geht es vor allem darum zuzuhören, Aufmerksamkeit entgegen zu bringen und dem Klienten gegenüber Empathie zu zeigen (Bluckert 2005a).

Ein wichtiger Aspekt in dem sich die Beziehung zwischen Coach und Therapeut gegenüber dem Klienten unterscheidet, ist beispielsweise, dass Coaching eher zukunftsorientiert und Therapie eher vergangenheitsbasiert ist (Bluckert, 2005a). Dies bedeutet, dass ein Coach viel motivierender und zielgerichteter agieren kann, als dies ein Therapeut tun könnte. Im Gegensatz zu Coaching erfordert Therapie eine tiefergehende Behandlung emotionaler Probleme (Greif 2008). Auch geht es im Unterschied zur Therapie bei den Reflexionen im Coaching eher um soziale und organisationale Themen, die durch Problemreflexionen präsenter sind (Greif et al. 2018). Obgleich es Unterschiede zwischen Coaching und Therapie gibt, so steht der gemeinsame Fokus des interpersonellen Umgangs und der gemeinsamen Interaktion vornehmlich im Mittelpunkt. Wir vertreten daher den Gesichtspunkt, dass die Coaching Interaktion und Beziehungsgestaltung von therapeutischen Befunden profitieren kann und die Ergebnisse nutzen sollte.

Im Folgenden gilt es darzustellen, wie die emotionalen Empathie, definiert als die Fähigkeit, eine Emotion nachempfinden zu können sowie die kognitiven Empathie beschrieben als die Fähigkeit, eine Emotion nachvollziehen zu können, gemessen werden können.

\section{Messung von Empathie}

Obwohl Empathie auch heute noch nicht einheitlich definiert ist (für einen Überblick siehe hierzu Will et al. 2016), ist man sich im psychologischen (klinischen) Forschungskontext einig, dass Empathie gemessen werden kann (u.a. Davis 1983; Hogan 1969; Jolliffe und Farrington 2006).

Viele Erhebungen beziehen sich auf die TherapeutenKlienten-Beziehung und die Wirkung von Empathie innerhalb dieses klinischen Interaktionskontextes (z.B. Burns 
und Nolen-Hoeksema 1992; Erskine et al. 2013; Lambert und Barley 2001). Dabei können drei Zugänge der Messung unterschieden werden (basierend auf Greenberg et al. 2001):

\section{Klienten-Einschätzungen (Fremdeinschätzung)}

Die Einschätzung der Klienten wird innerhalb der Forschung genutzt, um die Wahrnehmung des Klienten bezogen auf die gezeigte Empathie des Therapeuten zu erfassen. Der am weitesten verbreitete empathiebasierte Fragebogen, der Klienten vorgelegt wird, ist dabei der Barrett-Lennard Relationship Inventory (BLRI) (Barrett-Lennard 1986; Elliott et al. 2011; Gurman 1977). Der BLRI gründet vornehmlich auf der Perspektive von Rogers (Empathie, Kongruenz, Wertschätzung, Unvoreingenommenheit) bezüglich der Therapeuten-KlientenBeziehung, bei welcher Empathie eine wichtige Rolle spielt und liefert einen guten Überblick über beziehungsbildende Faktoren. Der Klienten-Einschätzung wird besondere Bedeutung beigemessen, da die durch den Klienten wahrgenommene Empathie des Therapeuten den Therapieerfolg im Vergleich zu anderen Messarten am besten voraussagt (z. B. Bohart et al. 2002).

2. Therapeuten-Einschätzungen (Selbsteinschätzung)

Der Therapeut kann seine eigene Empathiefähigkeit natürlich auch selbst einschätzen. Frühere Überblicksartikel zeigen auf, dass Therapeuten-Einschätzungen kaum mit klientenbasierten oder beobachterbasierten Einschätzungen korrelierten und den Therapieerfolg weniger gut vorhersagen konnten (z.B. Barrett-Lennard 1981). Diese Form der Erhebung wird daher, sofern sie eingesetzt wird, mit anderen Erhebungsmöglichkeiten gekoppelt.

3. Beobachtereingeschätzte Empathie

Ein Beobachter schätzt bei dieser Form der Erhebung ein, wie Therapeut und Klient miteinander interagieren. Innerhalb der angewandten Forschung findet diese Form der Empathiemessung bisher wenig Beachtung (Greenberg et al. 2001). Die Form der von Beobachtern eingeschätzten Empathie mittels Videobeobachtung findet hingegen immer mehr Beachtung. So konnten beispielsweise Gassmann und Grawe (2006) fünf Wirkfaktoren für die Beziehung zwischen Therapeut und Klient (Therapeutische Beziehung mit u. a. Empathie, Problemaktualisierung, Ressourcenaktivierung, Motivationale Klärung und Problembewältigung) mittels Videobeobachtung nachweisen.

Auf den klinischen Kontext bezogen kann zusammenfassend geschlussfolgert werden, dass das Konstrukt Empathie für die Therapeuten-Klienten-Beziehung zumeist mithilfe von Selbstauskünften geschieht (Gerdes et al. 2010). Erste wenige Ausnahmen analysieren das Konstrukt mittels Videobeobachtung (z.B. Gassmann und Grawe 2006). Wie der aktuelle Stand der Empathieforschung im Coaching ist, wird im Folgenden dargelegt.

\section{Empathie im Coaching}

Die Beziehung zwischen Coach und Klient wird häufig als ein Erfolgsfaktor im Coaching genannt (Grant et al. 2010; Graßmann und Schermuly 2017). Im Rahmen der Beziehungsgestaltung, wird auch im Coachingkontext die Bedeutung von Empathie für die Coach-Klienten-Beziehung betont (z. B. Bluckert 2005; Gregory und Levy 2011; Gyllensten und Palmer 2007; Neukom et al. 2011; O'Broin und Palmer 2009; Palmer und McDowall 2010). Es wird angenommen, dass die empathische positive Unterstützung durch den Coach eine wichtige Grundlage für eine Weiterentwicklung des Selbstwertgefühls des Klienten und des Erkennens seiner Bedürfnisse zu sein scheint (Bachkirova 2011). Dass empathisches Verhalten nicht nur innerhalb der Psychotherapie wichtig ist (Rogers 1959), sondern auch im Coaching, zeigen Stober und Grant (2006), die versuchen, die Basisvariablen, die auf Rogers zurückgehen, auf den Coachingkontext zu übertragen. Neben (1) positiver Wertschätzung und (2) Echtheit und Kongruenz wird hier (3) Empathie als eine der Grundlagen für die Therapeuten beziehungsweise Coach-Klienten-Interkation angeführt. Empathie ist hier sowohl als kognitive Empathie aufgeführt, die sich im Verhalten ausdrückt, als aber auch als emotionale Empathie.

Um bei dem Klienten eine Verhaltensänderung hervorzurufen, sollte die Beziehungsqualität zwischen Therapeut beziehungsweise Coach und Klient vom Klienten als empathisch bewertet werden. Eine empathische Beziehung von Coach und Klient ist demnach durch ausgedrücktes Coachverständnis charakterisiert und basiert auf unterstützenden Aussagen, die dem Klienten helfen sein Coaching Ziel zu erreichen. Auch Grawe (1998) hat basierend auf der Psychotherapieforschung ein Modell entwickelt, welches sich auf den Coachingkontext übertragen lässt. Neben weiteren Wirkfaktoren (Problemaktualisierung, Ressourcenaktivierung, Motivationale Klärung, Problembewältigung) wird auch hier die therapeutische Beziehung, charakterisiert durch empathisches Verhalten, als wichtiger Wirkfaktor im Coaching angeführt. Aufbauend auf den Arbeiten von Grawe hat auch Behrendt (2004, 2006) versucht, das therapeutische Erfolgsfaktorenmodell im Coachingkontext abzubilden. Empathie spielte bei dieser Untersuchung allerdings nur eine untergeordnete Rolle, da Behrendt sich vornehmlich auf die Haupterfolgs-Wirkfaktoren von Grawe (Problemaktualisierung, Klärung, Problembewältigung und Ressourcenaktivierung) stützte.

Neben Behrendt hat auch Greif (2008) sieben Coaching Wirkfaktoren (Wertschätzung und emotionale Unterstüt- 
zung, Affektaktivierung und Kalibrierung, ergebnisorientierte Problemreflexion, ergebnisorientierte Selbstreflexion, Zielklärung, Ressourcenaktivierung und Umsetzungsunterstützung) basierend auf Grawe herausgearbeitet. Auch bei diesen wird die wichtige Rolle der Coachempathie als ein wertvoller Wirkfaktor betont (hierbei ist Empathie als emotionale Unterstützung anzusehen). Die Empathie des Coaches zeigt sich im Verhalten. Der Coach soll dem Klienten neben wertschätzendem Verhalten auch emotionale (empathische) Unterstützung entgegenbringen (Greif et al. 2012).

Im Folgenden wird der Frage nachgegangen, wie die Rolle der Empathie im Coaching bislang empirisch untermauert werden konnte.

\section{Empirische Befunde von Empathie im Coaching}

Durch Schlagworteingabe mit Fokus auf dem Wort Empathie wurden die folgenden empirischen Befunde von Empathie im Coaching bei psycinfo (APA) und google scholar gefunden und dienen als Basis für die Analysen.

O'Broin und Palmer (2009) betonen, dass mehr Forschung zu Empathie und ihrer Wirkung auf die Arbeitsbeziehung nötig ist, um zu verstehen, was Coaching effektiv macht und wie die Coachingpraxis in der Zukunft vorangetrieben werden kann. Die wenigen empirischen Untersuchungen liefern bisher keine eindeutigen Erkenntnisse zur Bedeutung von Empathie im Coaching. So kann bis heute nur vereinzelt eine Differenzierung zwischen emotionaler und kognitiver Empathie im Coaching gefunden werden.

Baron und Morin (2009) untersuchten den Zusammenhang zwischen allgemeiner Empathie, und der Arbeitsbeziehung zwischen Coach und Klient empirisch. Bei den Coaches handelt es sich um Manager, die ihre Mitarbeiter bei der Entwicklung unterstützen sollten. Die Autoren analysierten Empathie nicht als eigenständiges Konstrukt, sondern als Konglomerat mit weiteren beziehungsbildenden Fähigkeiten wie Respekt, Vertrauen, Präsenz und Verfügbarkeit als eine Form von spezifischen Coachingfähigkeiten. Zwar konnten ihre Ergebnisse die Bedeutung der beziehungsbildenden Fähigkeiten für den Beziehungsaufbau zwischen Coach und Klient nicht untermauern. Jedoch zeigten sie die wichtige Bedeutung der Empathie für den Coach und den Klienten als Konglomerat von beziehungsbildenden Fähigkeiten auf. Baron und Morin spezifizieren die einzelne Operationalisierung von der gemessenen Empathie in ihrem Artikel nicht näher, beziehen sich in der Herleitung aber stark auf eine breite Literaturanalyse, in der sie Empathie erwähnen, aber nicht drauf eingehen, ob es sich um emotionale oder kognitive Empathie handelt.
In einer weiteren Studie analysierten Gregory und Levy (2011), inwiefern die gezeigte Empathie des Vorgesetzten (durch den Mitarbeiter wahrgenommen und eingeschätzt) als Mediator dafür fungiert wie, die selbstberichtete emotionalen Intelligenz des Vorgesetzten und die durch den Klienten berichtete Beziehungsqualität in Relation zueinander stehen. Sie haben Empathie als Interaktive Empathie operationalisiert, welche sofern sie wenig vorhanden ist in Relation zu Machiavellismus stehen kann. Ein Beispielitem ist „Mein Vorgesetzter fühlt die Emotionen, die ich erwarte." Die Mediationshypothese wurde nicht signifikant. Dies ist kein überraschendes Ergebnis, da wir aus neueren Erkenntnissen aus dem Coachingkontext wissen, dass es im Coaching nicht auf das Nachempfinden der Emotionen der Klienten durch die Coaches ankommt, sondern darauf, dass ein Coach sich empathisch verhält und dem Klienten zeigt, dass er sein Anliegen verstehen kann (Will et al. 2016). Zudem nimmt die Rolle des Vorgesetzten als Coach hier eine Sonderfunktion ein, die in der Analyse berücksichtigt werden sollte, da Mitarbeiter und Führungskraft in einem abhängigen Verhältnis zueinander stehen.

Weitere Studien konnten die Bedeutung von Empathie im Coachingkontext eindeutiger nachweisen, da sie fanden, dass eine positive Einschätzung des Klienten hinsichtlich der Empathie des Coaches oftmals mit positiven Coachingergebnissen zusammenhängt (z.B. de Haan et al. 2011).

Alle von uns recherchierten und gesichteten empirischen Studien zum Thema Empathie im Coaching erhoben das Konstrukt durch Fragebogenmethoden oder durch Selbstauskünfte. Des Weiteren wurde nicht zwischen emotionaler und kognitiver Empathie unterschieden. Wie wir weiter oben jedoch erläutert haben, ist besonders die kognitive Empathie bedeutend für Coachings, da ein Coach über das Verhalten sein Empathieverständnis dem Klienten vermitteln kann. Für den Coachingkontext ist das entgegengebrachte Verständnis gegenüber dem Klienten ausgedrückt durch kognitive Empathie zentraler als das emotionale Nachempfinden (emotionale Empathie) können.

Dem Ruf nach mehr prozessbasierter Forschung im Coaching (Feldman 2005; Möller und Kotte 2011), konnte bisher zwar durch eine stärker zunehmende Anzahl an interaktionsbasierten Studien (u. a. Behrendt 2004, 2006; Gessnitzer und Kauffeld 2015; Gessnitzer et al. 2016; Ianiro et al. 2013; Ianiro et al. 2015) nachgegangen werden. Der Frage nach Empathie als besonderer Wirkfaktor im Coaching und als prozessbeeinflussende Variable ging bisher jedoch nur eine Studie im Coachingkontext nach.

In einer eigenen Studie wurden 19 auf Video aufgezeichnete Coaching Dyaden mithilfe eines multimethodalen Forschungsdesigns (Kopplung aus Fragebogen Einsatz mit beobachtungsbasierten Daten) untersucht und die vom Coach gezeigte Empathie bezogen auf die Wahrnehmung des Klienten analysiert (Will et al. 2016). Die gezeigte Empathie 
ist in dieser Studie als kognitive Empathie operationalisiert worden, die sich im Verhalten äußert und durch den Klienten beurteilt wird. Ein Beispielitem der Fragebogenskala ist „Mein Coach versucht meine Sichtweise zu verstehen“, was deutlich die kognitive Komponente einklammert, hingegen die emotionale Empathie ausklammert, da dies im Coaching nicht fokussiert wird und es das Ziel vom Coach ist, den Klienten bestmöglich unterstützen und seinen Standpunkt nachvollziehen zu können. Weiterhin ist die kognitive Empathie durch Verhaltensweisen wie Paraphrasieren und Gefühle des Gegenübers ansprechen operationalisiert worden. Die kognitive Empathie kann folglich als verbale Unterstützung gegenüber dem Klienten von Coachseite erkannt werden. Die Ergebnisse zeigten unter anderem, dass vom Coach gezeigtes Paraphrasieren (als ein Teil von kognitiver Empathie) dazu betragen kann, vom Klienten als empathischer wahrgenommen zu werden. Diese Untersuchung von gezeigter Empathie im Coaching mittels Videobeobachtung kann als erster Ausgangspunkt für weitere prozessbasierte Forschung angesehen werden. Denn es ist notwendig, das Konstrukt der Empathie im Coaching gezielt zu untersuchen, um die Frage nach Wirkfaktoren zur Steigerung der Beziehungsqualität oder Verbesserung der Coachingergebnisse beantworten zu können, da wir aus dem Therapiekontext wissen, dass Empathie ein beziehungsbildender Faktor ist.

Darüber hinaus betont Greif (2011), dass es von elementarer Bedeutung für die Coachingforschung ist, sich mehr prozessbasierten Daten zu widmen. Statt sich weiterhin ausschließlich Prä- und/oder Post-Perspektiven zu bedienen, muss das direkte Verhalten in der reellen Situation erfasst werden. Da es sich bei reellen Verhaltensbeobachtungen um objektivere Daten als bei Selbstauskünften handelt, muss gerade im Coaching vermehrt der Fokus auf diese Erhebungsmethode gelegt werden. Erste Versuche, bei denen prozessbasierte Ansätze im Coaching eingesetzt wurden, zeigten, dass zum einen sowohl Empathie als generelles Thema mehr in den Coachingbereich einfließen sollte, zum anderen aber auch, dass die Wirkung der gezeigten Empathie des Coaches auf den Klienten eine signifikante Rolle einnimmt. Im Gegensatz zum klinischen Kontext wird hier jedoch explizit noch nicht zwischen den jeweiligen Empathieformen unterschieden. So wurde auch der BLRI bisher noch nicht in einer Coachingstudie angewandt.

\section{Verhaltensbeobachtung von Empathie im Coaching durch Videoanalysen}

Die Möglichkeit der Selbstauskunft bezüglich der eingeschätzten Empathie kann wichtige Ergebnisse für Coachingsitzungen liefern und ist eine effiziente Form der Erhebung. Dennoch handelt es sich bei Selbstauskünf- ten oftmals nur um eine retrospektive Einschätzung einer vorherigen Situation (Baumeister et al. 2007). Die Videoanalyse durch einen Beobachter kann hier unmittelbarere und objektivere Daten liefern. So kann man als Beobachter vor allem die kognitive Empathie als sich im Verhalten wiederspiegelndes Konstrukt erkennen. Neben Paraphrasieren könnten weitere Verhaltensanker darauf schließen, dass kognitive Empathie sich im Verhalten zeigt, z. B. durch wertschätzende Äußerungen oder durch Lob.

Ein mögliches beispielhaftes Instrument, mit welchem bereits kognitive Empathie in der publizierten eigenen Studie durch Videoanalyse untersucht wurde, ist act4consulting (Hoppe und Kauffeld 2010; Hoppe 2013). Act4consulting fußt auf dem für Teamprozesse basierten Interaktionstool act4teams (z. B. Kauffeld 2006; Kauffeld und Meyers 2009; Kauffeld und Montasem 2009; Kauffeld und Lehmann-Willenbrock 2012; Kauffeld et al. 2018; Meinecke und Kauffeld 2016; Schulte et al. 2013). Das aus 46 Kategorien bestehende Instrument act4consulting wurde explizit dafür entwickelt, feinkörnige zeitliche verbale Prozesse basierend auf Moment-zu-Moment Dynamiken auf dem Konversationslevel zu untersuchen (vgl. Kauffeld et al. 2018). Für die präzise Interaktionsanalyse können die jeweiligen Interaktionsmikrosequenzen in kleinste Sinneinheiten unterteilt werden. Für diese Art der Analyse muss ein Kodierer eine Ausbildung mithilfe der Benutzung dieses Instruments durchlaufen.

Instrumente wie act4consulting wurden bereits erprobt und häufig in Erhebungen eingesetzt (z.B. Gessnitzer und Kauffeld 2015; Gessnitzer et al. 2016; Will et al. 2016). Aus diesem Grund sollte sich zukünftige Forschung, die sich den Bereich Empathie im Coaching ansehen möchte, der Videoanalyse bedienen oder diese in Kombination mit Fragebögen einsetzen. Nur so kann ein umfassenderes Bild über das Konstrukt Empathie in der Coachinginteraktion gewonnen werden und die Forschungslücke, ähnlich wie in der psychotherapeutischen Forschung, kontinuierlich geschlossen werden.

\section{Limitationen, Schlussfolgerungen und Perspektiven für zukünftige Forschung von Empathie im Coaching}

Das Ziel dieses Beitrags ist es, den aktuellen Forschungsstand von Empathie im Coaching vorzustellen und dabei auf dem psychotherapeutischen Kontext aufzubauen und die ersten publizierten Ergebnisse im Coaching zu präsentieren. Wie gezeigt werden konnte, steht die Forschung zum Thema Empathie hier noch am Anfang. Zwar gibt es erste Studien, die versuchen theoretisch begründet die psychotherapeutischen Modelle und Erkenntnisse auf den Coachingkontext zu übertragen (z.B. Behrendt 2004, 2006; Greif 
2011; Stober und Grant 2006; Stober et al. 2006), doch empirische Studien mit dem Fokus auf Empathie fehlen bisher fast gänzlich. Dies scheint bei der hohen allgemein angemerkten Relevanz der Empathie innerhalb der publizierten Coachingstudien verwunderlich. Eine Möglichkeit könnte sein, dass Empathie häufig nicht als eine einzelne Variable innerhalb von Coachinguntersuchungen angesehen wird, sondern oftmals als ein Teil von beispielsweise beziehungsbildenden Variablen verstanden wird (vgl. Baron und Morin 2009). Dass man Empathie allerdings als Einzelvariable messen sollte, unterstützen die Erkenntnisse aus dem Psychotherapiekontext, da Empathie hier als fundamentales Element einer Beziehung identifiziert wurde. Die Arbeiten von Rogers dienten hier in ersten theoretischen Herleitungen bereits als Basismodell für etwaige Forschungsarbeiten im Coaching. Ähnlich wie in der psychotherapeutischen Forschung werden die wenigen Erhebungen, die sich mit Empathie im Coaching befassen, per Fragebogen gemessen und nur in geringem Maße mithilfe von Videobeobachtungen oder multimethodalen Ansätzen (Will et al. 2016) erforscht. Greif und Kollegen (2012) schätzen, dass die Coachingforschung an diesem Punkt noch in den Anfängen steckt, da Beobachtungsstudien sehr zeitintensiv und große Stichproben kaum zu erzielen sind. Um Empathie jedoch im Coachingkontext richtig messen zu können, sollte man zum einen von bereits etablierten Disziplinen, wie der Psychotherapie, lernen. Zum anderen sich jedoch auch weiterer Messmöglichkeiten wie Beobachtungsinstrumenten bedienen, da hier reelle Verhaltensbeobachtungen ermöglicht werden können und die Interaktion objektiv beobachtet und analysiert werden kann. Auch könnte der nicht passgenaue Einsatz von allgemeinen Empathie-Fragebögen für nicht signifikante Ergebnisse als Erklärung herangezogen werden. Die Unterscheidung zwischen emotionaler und kognitiver Empathie sollte für den Coachingkontext präsenter gemacht werden, da es sich hierbei um inhaltlich unterschiedliche Konstruktteile handelt. Innerhalb einer Coachingsitzung sollte der Hauptfokus des Coachs auf dem Klienten und dem Verstehen sowie Unterstützen seines Coachinganliegens basieren. Hierfür erscheint die kognitive Empathie als Basis grundlegender zu sein, als die emotionale Empathie (Bluckert 2005; Will et al. 2016). Zukünftige Untersuchungen sollten sich also speziell der kognitiven Empathie als potenzieller Messmöglichkeit von Empathie bedienen und sich diese als sich im Verhalten wiederspiegelnde Variable analysieren.

Weiterhin sollte den einzelnen Kontexten, in denen Empathie im Coaching gemessen wird, mehr Aufmerksamkeit geschenkt werden. So sollte beispielsweise der Fokus auf unterschiedliche Coaching Settings und den einzelnen „Coachs“, die das Coaching durchführen, liegen. Der Unterschied zwischen einem Coaching innerhalb eines Unternehmens mit einem hierarchisch höher geranktem Coach und einem externen oder hierarchisch ähnlichem Coach (ggf. sogar außerhalb des Unternehmens) wird höchstwahrscheinlich anders ausfallen, da hier andere Einflussfaktoren eine Rolle spielen. Auch würde mitunter ein Manager, der in seiner Hauptjobrolle Führungskraft ist sich seinem Mitarbeiter gegenüber anders verhalten als ein externer, unternehmensunabhängiger Coach, der nicht vorrangig z.B. das Unternehmenswachstum berücksichtigen muss. Erst wenn man unterschiedliche Kontexte untersucht hat, kann man allgemeinere Aussagen über den Einsatz von Empathie im Coaching treffen. Auch könnte man die im klinischen Kontext berichteten Therapeuten-Effekte (z.B. Lambert 2013) mit dem Coachingkontext vergleichen, um hier analysieren zu können, inwiefern diese Effekte eine Auswirkung auf beispielsweise die Beziehung zwischen Coach und Klient haben. Zusätzlich könnte man analysieren inwiefern die vom Coach gezeigte Empathie sich von anderen Effekten unterscheidet.

Coachingfähigkeiten, wie z.B. das Paraphrasieren (vorherige Aussagen des Klienten in eigenen Worten zusammenfassen und wiedergeben) als ein Teil von kognitiver Empathie (vgl. Will et al. 2016), können in Coaching-Weiterbildungen gezielt erlernt werden.

Neben dem Konstrukt der Empathie als wichtiger Einflussfaktor für Coachings, sollte sich zukünftige Forschung noch weitere Faktoren, wie beispielsweise Emotionale Intelligenz oder interpersonale Kompetenz des Coachs, angucken, um besser verstehen zu können wie Coaching wirkt. Zusätzlich zu der verbalen Empathiemessung, könnte man sich zusätzlich noch auf nonverbale Merkmale beziehen, da diese für ein allumfassendes Gesamtbild der kognitiven Empathie behilflich sein können. Auch ein spannender Forschungspunkt für zukünftige Untersuchungen wäre die Frage inwiefern Perspektivübernahme in Bezug gesetzt werden kann zu Empathie und welche Rolle hier die kognitive Empathie spielt.

Der jedoch wohl wichtigste Punkt wird in zukünftigen Empathiemessungen die richtige Operationalisierung des Konstrukts sein, da sich kognitive Empathie anders zeigt, als emotionale Empathie.

Es kann zusammenfassend festgehalten werden, dass der Bedeutung von Empathie für die Beziehungsgestaltung zwischen Coach und Klient mehr Aufmerksamkeit entgegengebracht werden sollte. Empathie zur Förderung und Stärkung einer Coach-Klienten-Beziehung als zentraler Wirkfaktor scheint sowohl für Forscher, deren Ziel es ist, weiterhin Licht ins Dunkel der Frage zu bringen, wie Coaching eigentlich wirkt, als auch für Praktiker unverzichtbar zu sein. 


\section{Praktische Implikationen}

Da sich die kognitive Empathie im Verhalten manifestiert, ist es besonders wichtig, sich als praktizierender Coach darüber klar zu werden, wann man empathisches Verhalten einsetzt, um den Klienten zu unterstützen und ihm bestmöglich gegenüberzutreten. Coaches sollten sich nicht nur auf ihr Gefühl und ihre Erfahrungen verlassen, sondern theoriegeleitet arbeiten und validierte Instrumente oder Forschungserkenntnisse berücksichtigen und in ihre tägliche Arbeit einbauen (Grant et al. 2010). Nur so kann die Qualität von Coaching langfristig gewährleistet werden. Ein mögliches Instrument, welches für die Erhebung der sich im Verhalten gezeigten Empathie wichtig sein könnte, ist das oben erwähnte Instrument act4consulting (u. a. Hoppe 2013). Für einen Coach bedeutet die Nutzung des Instrumentes, dass er sich im Nachgang an eine Coachingsitzung seine auf Video aufgenommenen empathischen Verhaltensweisen ansehen kann. Als Beobachter ergibt sich die Möglichkeit, zu erkennen, wann ein Coach empathisch interagiert hat und wo ein Coach sich gegebenenfalls hätte anders verhalten sollen, da der Klient beispielsweise nicht positiv auf die gezeigte Verhaltensweise reagierte. Im zweiten Schritt kann dann analysiert werden, wie ein Coach sich an gewissen Stellen bestmöglich empathisch verhalten hätte oder wo ein gewisses empathisches Verhalten hätte vertieft werden sollen. Insbesondere für Weiterbildungsmaßnahmen und Trainings von angehenden Coaches bietet sich die Reflektion und Nachbearbeitung einer Coachingsitzung mithilfe des act4consulting Instruments an.

Zusätzlich kann auch das von Rogers im therapeutischen Kontext begründete und hier angeführte aktive $\mathrm{Zu}$ hören oder die oftmals auch bereits im Coachingkontext erwähnte Wertschätzung (z.B. Greif et al. 2012; Rogers 1951) als weitere potenzielle Empathie unterstützende Verhaltensweise mithilfe des Instruments erfasst werden.

Was bedeuten die bisherig gefundenen Ergebnisse für praktizierende Coaches? An erster Stelle ist es wichtig, dass ein Coach sich darüber bewusst ist, dass seine Selbsteinschätzung nicht immer identisch ist mit jener des Klienten. Um den Klienten bestmöglich bei seinem Prozess im Coaching zu unterstützen, ist es wichtig, mit einer gewissen empathischen Grundhaltung in das Coaching zu gehen. Ein Coach sollte dem Klienten zeigen, dass er ihn und seine Anliegen versteht und sich ihm gegenüber empathisch verhalten. Eine Möglichkeit ist beispielsweise die Form des Paraphrasierens. Durch diese gezeigte empathische Grundhaltung kann das Handlungsinteresse des Klienten verstärkt werden und seine Impulse besser zum Ausdruck kommen. Auch sollte ein Coach das Umfeld eines Coachings berücksichtigen und sich seiner gegebenenfalls hierarchischen Rolle, als beispielsweise Führungskraft, bewusst sein. Generell sollte ein Coach in seinem Verhalten offen sein und die bisherig gewonnen empathiebasierten Erkenntnisse in seiner Arbeit berücksichtigen. Abschließend kann geschlussfolgert werden, dass Empathie nicht nur in der Forschung an Präsenz gewinnen sollte, sondern sich auch praktizierende Coaches der wichtigen Rolle von Empathie bewusst werden und diese im Coachingprozess zeigen sollten.

Interessenkonflikt T. Will und S. Kauffeld geben an, dass kein Interessenkonflikt besteht.

Open Access Dieser Artikel wird unter der Creative Commons Namensnennung 4.0 International Lizenz (http://creativecommons.org/ licenses/by/4.0/deed.de) veröffentlicht, welche die Nutzung, Vervielfältigung, Bearbeitung, Verbreitung und Wiedergabe in jeglichem Medium und Format erlaubt, sofern Sie den/die ursprünglichen Autor(en) und die Quelle ordnungsgemäß nennen, einen Link zur Creative Commons Lizenz beifügen und angeben, ob Änderungen vorgenommen wurden.

\section{Literatur}

Anderson, T., Ogles, B.M., Patterson, C.L., Lambert, M.J., \& Vermeersch, D. A. (2009). Therapist effects: facilitative interpersonal skills as a predictor of therapist success. Journal of Clinical Psychology, 65, 755-768. https://doi.org/10.1002/jclp.20583.

Bachkirova, T. (2011). Developmental coaching: working with the self: working with the self. UK: McGraw-Hill.

Baron, L., \& Morin, L. (2009). The coach-coachee relationship in executive coaching: a field study. Human Resource Development Quarterly, 20(1), 85-106. https://doi.org/10.1002/hrdq.20009.

Baron-Cohen, S., \& Wheelwright, S. (2004). The empathy quotient: an investigation of adults with Asperger syndrome or high functioning autism, and normal sex differences. Journal of Autism and Developmental Disorders, 34(2), 163-175. https://doi.org/10. 1023/B:JADD.0000022607.19833.00.

Barrett-Lennard, G.T. (1981). The empathy cycle: refinement of a nuclear concept. Journal of Counseling Psychology, 28(2), 91-100.

Barrett-Lennard, G. T. (1986). The Relationship Inventory now: Issues and advances in theory, method, and use. In L. S. Greenberg \& W. M. Pinsof (Hrsg.), Guilford clinical psychology and psychotherapy series. The psychotherapeutic process: a research handbook (S. 439-476). New York: Guilford.

Baumeister, R.F., Vohs, K. D., \& Funder, D. C. (2007). Psychology as the science of self-reports and finger movements: whatever happened to actual behavior? Perspectives on Psychological Science, 2, 396-403. https://doi.org/10.1111/j.1745-6916.2007.00051.x.

Behrendt, P. (2006). Wirkung und Wirkfaktoren von psychodramatischem Coaching - Eine experimentelle Evaluationsstudie. Zeitschrift für Psychodrama und Soziometrie, 5(1), 59-87. https://doi. org/10.1007/s11620-006-0006-6.

Behrendt, P. (2004). Wirkfaktoren im Psychodrama und Transfercoaching. Unveröffentlichte Diplomarbeit, Albert-Ludwigs-Universität Freiburg.

Bluckert, P. (2005). Critical factors in executive coaching - the coaching relationship. Industrial and Commercial Training, 37, 336-340. https://doi.org/10.1108/00197850510626785.

Bluckert, P. (2005a). The similarities and differences between coaching and therapy. Industrial and Commercial Training, 37, 91-96. https://doi.org/10.1108/00197850510584241.

Bohart, A.C., Elliott, R., Greenberg, L.S., \& Watson, J.C. (2002). Empathy. In J.C. Norcross (Hrsg.), Psychotherapy relationships 
that work: Therapist contributions and responsiveness to patients (S. 89-108). New York: Oxford University Press.

Burns, D. D., \& Nolen-Hoeksema, S. (1992). Therapeutic empathy and recovery from depression in cognitive-behavioral therapy: a structural equation model. Journal of Consulting and Clinical Psychology, 60(3), 441-449. https://doi.org/10.1037/0022-006X.60. 3.441 .

Dadds, M.R., Hunter, K., Hawes, D. J., et al. (2008). A measure of cognitive and affective empathy in children using parent ratings. Child Psychiatry and Human Development, 39(2), 111-122. https://doi.org/10.1007/s10578-007-0075-4.

Davis, M.H. (1983). Measuring individual differences in empathy: evidence for a multidimensional approach. Journal of Personality and Social Psychology, 51(2), 167-184.

Eisenberg, N., \& Miller, P.A. (1987). The relation of empathy to prosocial and related behaviors. Psychological Bulletin, 101(1), 91-119. https://doi.org/10.1037/0033-2909.101.1.91.

Elliott, R., Bohart, A. C., Watson, J. C., \& Greenberg, L. S. (2011). Empathy. In J. Norcross (Hrsg.), Psychotherapy relationships that work (2. Aufl. S. 132-152). New York: Oxford University Press.

Erskine, R., Moursund, J., \& Trautmann, R. (2013). Beyond empathy: a therapy of contact-in relationships. New York: Routledge.

Feldman, D.C. (2005). Executive coaching: a review and agenda for future research. Journal of Management, 31(6), 829-848.

Feldman, D.C., \& Lankau, M. J. (2005). Executive coaching: a review and agenda for future research. Journal of Management, 31(6), 829-848.

Gassmann, D., \& Grawe, K. (2006). General change mechanisms: the relation between problem activation and resource activation in successful and unsuccessful therapeutic interactions. Clinical Psychology and Psychotherapy, 13(1), 1-11.

Gerdes, K.E., Segal, E.A., \& Lietz, C.A. (2010). Conceptualising and measuring empathy. British Journal of Social Work, 40(7), 2326-2343. https://doi.org/10.1093/bjsw/bcq048.

Gessnitzer, S., \& Kauffeld, S. (2015). The working alliance in coaching: why behavior is the key to success. Journal of Applied Behavioral Science, 51(2), 177-197. https://doi.org/10.1177/ 0021886315576407.

Gessnitzer, S., Schulte, E.M., \& Kauffeld, S. (2016). "I am going to succeed": the power of self-efficient language in coaching and how coaches can use it. Consulting Psychology Journal: Practice and Research, 68(4), 294-312. https://doi.org/10.1037/ cpb0000064.

Grant, A., Passmore, J., Cavanagh, M., \& Parker, H. (2010). The state of play in coaching today: a comprehensive review of the field. International Review of Industrial and Organizational Psychology, 25, 125-167. https://doi.org/10.1002/9780470661628.

Graßmann, C., \& Schermuly, C.C. (2017). The role of neuroticism and supervision in the relationship between negative effects for clients and novice coaches. Coaching: An International Journal of Theory, Research and Practice, 11(1), 74-88. https://doi.org/10.1080/ 17521882.2017.1381755.

Grawe, K. (1998). Psychologische Therapie. Göttingen: Hogrefe.

Greenberg, L. S., Watson, J. C., Elliott, R., \& Bohart, A. C. (2001). Empathy. Psychotherapy, 38(4), 380-384.

Gregory, J.B., \& Levy, P.E. (2011). It's not me, it's you: a multilevel examination of variables that impact employee coaching relationships. Consulting Psychology Journal: Practice and Research, 63(2), 67-88. https://doi.org/10.1037/a0024152.

Greif, S. (2008). Coaching und ergebnisorientierte Selbstreflexion: Theorie, Forschung und Praxis des Einzel- und Gruppencoachings. Göttingen: Hogrefe.

Greif, S. (2011). Gesundheits- und Stressmanagementcoaching. In E. Bamberg, A. Ducki \& A.-M. Metz (Hrsg.), Handbuch Gesundheitsförderung und Gesundheitsmanagement in der Arbeitswelt Ein Handbuch (S. 341-369). Göttingen: Hogrefe.

Greif, S. (2013). Putting goals to work in coaching: the complexities of implementation. In D. Megginson \& D. Clutterbuck (Hrsg.),
Beyond goals: effective strategies for coaching and Mentoring. Farnham: Gower.

Greif, S., Schmidt, F., \& Thamm, A. (2012). Warum und wodurch Coaching wirkt. Organisationsberatung, Supervision, Coaching, 19, 375-390. https://doi.org/10.1007/s11613-012-0299-4.

Greif, S., Möller, H., \& Scholl, W. (2018). Coachingdefinitionen und -konzepte. In Handbuch Schlüsselkonzepte im Coaching (S. 1-9). Berlin, Heidelberg: Springer.

Gurman, A. S. (1977). The patient's perception of the therapeutic relationship. In A. S. Gurman \& A. M. Razin (Hrsg.), Effective psychotherapy: a handbook of research (S. 503-543). New York: Pergamon.

Gyllensten, K., \& Palmer, S. (2007). The coaching relationship: an interpretative phenomenological analysis. International Coaching Psychology Review, 2(2), 168-177.

de Haan, E., Culpin, V., \& Curd, J. (2011). Executive coaching in practice: what determines helpfulness for clients of coaching? Personnel Review, 40, 24-44. https://doi.org/10.1108/ 00483481111095500.

Hall, J.A., \& Bernieri, F. J. (2001). Interpersonal sensitivity: theory and measurement. New York: Psychology Press.

Hall, D. T., Otazo, K.L., \& Hollenbeck, G.P. (1999). Behind closed doors: what really happens in executive coaching. Organizational Dynamics, 27(3), 39-53. https://doi.org/10.1016/S00902616(99)90020-7.

Hatfield, E., Rapson, R.L., \& Le, Y.L. (2009). Emotional contagion and empathy. In J. Decety \& W. Ickes (Hrsg.), The social neuroscience of empathy (S. 19-30). Cambridge: MIT Press.

Hogan, R. (1969). Development of an empathy scale. Journal of Consulting and Clinical Psychology, 33, 307-316. https://doi.org/10. 1037/h0027580.

Hoppe, D., \& Kauffeld, S. (2010). Positive Gefühle zählen - Ein Analyseverfahren zeigt, was in Beratungen wirkt. Wirtschaftspsychologie aktuell, 3, 34-37.

Hoppe, D. (2013). Was passiert in Beratungsgesprächen? Eine mikroanalytische Betrachtung von Berater-Klienten-Interaktionen in der Inhaltsberatung (Doktorarbeit). Technische Universität Braunschweig, Braunschweig, Germany.

Horvath, A. O., De Re, A. C., Flückiger, C., \& Symonds, D. (2011). Alliance in individual psychotherapy. Psychotherapy, 48(1), 9-16. https://doi.org/10.1037/a0022186.

Ianiro, P. M., \& Kauffeld, S. (2014). Take care what you bring with you: How coaches' mood and interpersonal behavior affect coaching success. Consulting Psychology Journal: Practice and Research, 66(3), 231-257. https://doi.org/10.1037/cpb0000012.

Ianiro, P. M., Schermuly, C. C., \& Kauffeld, S. (2013). Why interpersonal dominance and affiliation matter: an interaction analysis of the coach-client relationship. Coaching: An International Journal of Theory, Research and Practice, 6(1), 25-46. https://doi.org/10. 1080/17521882.2012.740489.

Ianiro, P. M., Lehmann-Willenbrock, N., \& Kauffeld, S. (2015). Coaches and clients in action: a sequential analysis of interpersonal coach and client behavior. Journal of Business and Psychology, 30, 435-456. https://doi.org/10.1007/s10869-014-9374-5.

Jolliffe, D., \& Farrington, D.P. (2006). Development and validation of the Basic Empathy Scale. Journal of Adolescence, 29(4), $589-611$.

Kauffeld, S. (2006). Kompetenzen messen, bewerten, entwickeln - Ein prozessanalytischer Ansatz für Gruppen. Stuttgart: Schaffer-Poeschel.

Kauffeld, S., \& Lehmann-Willenbrock, N. (2012). Meetings matter: effects of team meetings on team and organizational success. Small Group Research, 43, 130-158. https://doi.org/10.1177/ 1046496411429599.

Kauffeld, S., \& Meyers, R. (2009). Complaint and solution-oriented circles: interaction patterns in work group discussions. European Journal of Work and Organizational Psychology, 18, 267-294. https://doi.org/10.1080/13594320701693209. 
Kauffeld, S., \& Montasem, K. (2009). Ein Kompetenzmodell als Basis. Professionelle Video-Analyse im Coaching. Coaching-Magazin, 4, 44-49.

Kauffeld, S., Lehmann-Willenbrock, N., \& Meinecke, A.L. (2018). The Advanced Interaction Analysis for Teams (act4teams) coding scheme. In E. Brauner, M. Boos \& M. Kolbe (Hrsg.), The Cambridge handbook of group interaction analysis. New York: Cambridge University Press.

Kurtz, R.R., \& Grummon, D.L. (1972). Different approaches to the measurement of therapist empathy and their relationship to therapy outcomes. Journal of Consulting and Clinical Psychology, 39, $106-115$.

Lambert, M. J. (2013). Bergin and Garfield's handbook of psychotherapy and behavior change. New York: John Wiley \& Sons.

Lambert, M. J., \& Barley, D.E. (2001). Research summary on the therapeutic relationship and psychotherapy outcome. Psychotherapy: Theory, Research, Practice, Training, 38(4), 357-361.

Mehrabian, A., Young, A.L., \& Sato, S. (1988). Emotional empathy and associated individual differences. Current Psychology, 7(3), 221-240.

Meinecke, A.L., \& Kauffeld, S. (2016). Interaktionsanalyse in Gruppen: Anwendung und Herausforderungen. Gruppe. Interaktion. Organisation. (GIO), 47, 321-333. https://doi.org/10.1007/ s11612-016-0347-1.

Miller, P.A., \& Eisenberg, N. (1988). The relation of empathy to aggressive and externalizing/antisocial behavior. Psychological Bulletin, 103(3), 324-344.

Möller, H., \& Kotte, S. (2011). Die Zukunft der Coachingforschung. Organisationsberatung, Supervision, Coaching, 18(4), 445-456. https://doi.org/10.1007/s11613-011-0256-7.

Neukom, M., Schnell, K., \& Boothe, B. (2011). Die Arbeitsbeziehung im Coaching - ein Stiefkind der Forschung. Organisationsberatung, Supervision, Coaching, 18, 317-332. https://doi.org/10. 1007/s11613-011-0242-0.

Nicolai, J., Demmel, R., \& Hagen, J. (2007). Rating scales for the assessment of empathic communication in medical interviews (REM): Scale development, reliability, and validity. Journal of Clinical Psychology in Medical Settings, 14(4), 367-375. https:// doi.org/10.1007/s10880-007-9081-8.

Norcross, J.C., \& Wampold, B.E. (2011). Evidence-based therapy relationships: research conclusions and clinical practices. Psychotherapy, 48(1), 98-102.

O'Broin, A., \& Palmer, S. (2009). Co-creating an optimal coaching alliance: a cognitive behavioural coaching perspective. International Coaching Psychology Review, 4(2), 184-194.

Palmer, S., \& McDowall, A. (2010). The coaching relationship. London: Routledge.
Parra, A. (2013). Cognitive and emotional empathy in relation to five paranormal/anomalous experiences. North American Journal of Psychology, 15(3), 405-412.

Preston, S.D., \& Hofelich, A.J. (2011). The many faces of empathy: Parsing empathic phenomena through a proximate, dynamic-systems view of representing the other in the self. Emotion Review, $4(1), 1-10$.

Reik, T. (1948). Listening with the third ear. New York: Grove Press.

Reniers, R. L., Corcoran, R., Drake, R., Shryane, N. M., \& Völlm, B. A. (2011). The QCAE: a questionnaire of cognitive and affective empathy. Journal of Personality Assessment, 93(1), 84-95. https:// doi.org/10.1080/00223891.2010.528484.

Rogers, C.R. (1951). Client-centered therapy. Boston: HoughtonMifflin.

Rogers, C.R. (1957). The necessary and sufficient conditions of therapeutic personality change. Journal of Consulting Psychology, 21(2), 95-103. https://doi.org/10.1037/h0045357.

Rogers, C. R. (1959). A theory of therapy, personality and interpersonal relationships as developed in the client centered framework. In S. Koch (Hrsg.), Psychology: a study of a science: formulations of the person and the social context 3. Aufl. New York: McGrawHill.

Rogers, C.R. (2007). The necessary and sufficient conditions of therapeutic personality change. Psychotherapy: Theory, Research, Practice, Training, 44(3), 240-248. https://doi.org/10.1037/00333204.44.3.240.

Schulte, E. M., Fenner, T., \& Kauffeld, S. (2013). Nicht ohne Nebenwirkungen: Gesundheitsrisiko Meeting. Personal Quarterly, 2, $8-15$.

Smith, A. (1759). Theory of moral sentiments. London: A. Miller.

Smith, A. (2006). Cognitive empathy and emotional empathy in human behavior and evolution. The Psychological Record, 56, 3-21.

Spencer, H. (1870). The principles of psychology. London: Williams \& Norgate.

Stober, D.R., \& Grant, A. (2006). Toward a contextual approach to coaching models. In D. Stober \& A. M. Grant (Hrsg.), Evidencebased coaching handbook. New York: Wiley.

Stober, D. R., Wildflower, L., \& Drake, D. (2006). Evidence-based practice: A potential approach for effective coaching. International Journal of Evidence Based Coaching and Mentoring, 4(1), 1-

Will, T., Gessnitzer, S., \& Kauffeld, S. (2016). You think you are an empathic coach? Maybe you should think again. The difference between perceptions of empathy vs. empathic behaviour after a person-centered coaching training. Coaching: An International Journal of Theory, Research and Practice, 9(1), 53-68. https:// doi.org/10.1080/17521882.2016.1143023. 